\title{
HOMOTOPY GROUPS OF THE COMPLEMENTS TO SINGULAR HYPERSURFACES
}

\author{
BY A. LIBGOBER
}

In the early thirties $\mathrm{O}$. Zariski (cf. [6, Chapter 8]) discovered a relationship between the fundamental group of the complement to a plane curve in $\mathbf{C P}^{2}$ and the first Betti number of cyclic covers of $\mathbf{C P}^{2}$ branched over this curve. At the same time, he determined the precise relationship between this Betti number and the position of singularities of the branching locus. Here we shall describe the relationship between certain higher homotopy groups of the complement to a hypersurface in $\mathbf{C P}^{n+1}$ and Hodge numbers of cyclic covers of $\mathbf{C P}^{n+1}$ branched over this hypersurface, as well as their relation to the position of singularities of the branching locus.

Let $V_{d}^{n}$ be a hypersurface of degree $d$ in $\mathbf{C P}^{n+1}$. Let $k$ denote the dimension of the singular locus of $V_{d}^{n}$. We shall fix a generic hyperplane $H$ (i.e., transversal to the strata of the singular locus of $V_{d}^{n}$ ). Let $H_{n-k}$ denote a generic linear subspace of $\mathbf{C P}^{n+1}$ of dimension $n-k$. It follows from Zariski's theorem ([2]) and the computation of homotopy groups of the complement to a nonsingular hypersurface (cf. [1]) that

$$
\pi_{\imath}\left(\mathbf{C P}^{n+1}-V_{d}^{n}\right)=\pi_{\imath}\left(H_{n-k}-V_{d}^{n}\right)= \begin{cases}\mathbf{Z} / d & i=1, \\ 0 & i=2, \ldots, n-k-1,\end{cases}
$$

and

$$
\pi_{i}\left(\mathbf{C P}^{n+1}-\left(V_{d}^{n} \cup H\right)\right)= \begin{cases}\mathbf{Z} & i=1, \\ 0 & i=2, \ldots, n-k-1,\end{cases}
$$

Therefore the first homotopy group of $\mathbf{C P}^{n+1}-V_{d}^{n}\left(\operatorname{resp} . \mathbf{C P}^{n+1}-\left(V_{d}^{n} \cup H\right)\right)$ which can be affected by singularities of $V_{d}^{n}$ is $\pi_{n-k}$. From now on we shall consider $\pi_{n-k}\left(\mathbf{C P}^{n+1}-V_{d}^{n}\right)\left(\right.$ resp. $\left.\pi_{n-k}\left(\mathbf{C P}^{n+1}-\left(V_{d}^{n} \cup H\right)\right)\right)$ as $\mathbf{Z} / d$-module (resp. as $\mathbf{Z}$-module), where the action is the usual action of fundamental group on a homotopy group.

Proposition 1. Let $d \mathbf{Z}$ denote the subgroup of index $d$ in $\mathbf{Z}$. Then $\pi_{n-k}\left(\mathbf{C P}^{n+1}-V_{d}^{n}\right)$ is isomorphic to the submodule of invariants

$$
\pi_{n-k}\left(\mathbf{C P}^{n+1}-\left(V_{d}^{n} \cup H\right)^{\operatorname{Inv} d \mathbf{Z}}\right)
$$

as $\mathbf{Z} / d$-module.

Received by the editors August 7, 1984.

1980 Mathematics Subject Classification. Primary 14F20, 57M05, 14H20, 57M10, 14J17, 57M15. 
Let $H_{s}, s \in \mathrm{CP}^{1}$, be a generic pencil of hyperplanes in $\mathbf{C P}^{n+1}$, and let $S$ be a subset in $\mathrm{CP}^{1}$ corresponding to nongeneric elements in $H_{s}$. Then the homotopy type of $H_{s}-\left(V_{d}^{n} \cup H\right)$ is independent of $s$ for $s \notin S$. Let $e_{i}, i=1, \ldots,|S|$ be a system of loops in $\mathbf{C P}^{1}-S$ forming a basis of $\pi_{1}\left(\mathbf{C P}^{1}-S\right)$. The monodromy along $e_{i}$ defines the automorphism $E_{i}$ of $\left.\pi_{n-k}\left(H_{s}-V_{d}^{n} \cup H\right)\right)$. Let $g_{1}, \ldots, g_{T}$ be a system of generators of $\pi_{n-k}\left(H_{s}-\left(V_{d}^{n} \cup H\right)\right)$ as $\mathbf{Z}\left[t, t^{-1}\right]$-module. The following is an analog of a theorem of Van Kampen [5].

THEOREM 1. $\pi_{n-k}\left(\mathbf{C P}^{n+1}-\left(V_{d}^{n} \cup H\right)\right)$ is isomorphic as a $\mathbf{Z}\left[t, t^{-1}\right]$-module to the quotient of $\pi_{n-k}\left(H_{s}-\left(V_{d}^{n} \cup H\right)\right)$ by submodule generated by $E_{i}\left(g_{j}\right)-g_{j}$, $j=1, \ldots, T, i=1, \ldots,|S|$.

From now on we shall assume that $V_{d}^{n}$ is a Q-manifold.

Proposition 2. The homotopy group $\pi_{n-k}\left(\mathbf{C P}^{n+1}-\left(V_{d}^{n} \cup H\right)\right) \otimes \mathbf{Q}$ is a torsion $\mathrm{Q}\left[t, t^{-1}\right]$-module, i.e., is isomorphic to $\oplus_{i=1}^{l} \mathrm{Q}\left[t, t^{-1}\right] / \Delta_{i}$, where $\Delta_{i}$ are certain elements of $\mathrm{Q}\left[t, t^{-1}\right]$. Moreover $\Delta_{i}(1)=1$.

We call $P=\prod_{i=1}^{l} \Delta_{i}$ the $\mathrm{Q}\left[t, t^{-1}\right]$-order of $\pi_{n-k}$.

Let $\tilde{X}_{m}$ denote a desingularization of the $m$-fold cyclic cover of $\mathbf{C P}^{n+1}$ branched over $V_{n}^{d}$ and possibly the hyperplane $H$ (i.e., a desingularization of projective closure of the affine hypersurface $z_{0}^{m}=f\left(z_{1}, \ldots, z_{n+1}\right)$, where $f$ is an equation of $V_{d}^{n}$ ).

Proposition 3. Let $h^{n, 0}\left(\tilde{X}_{m}\right)=\operatorname{dim} H^{n}\left(\tilde{X}_{m}, O_{\tilde{X}_{m}}\right)$. Then $h^{n, 0}\left(\tilde{X}_{m}\right)$ does not exceed the sum of the numbers of common roots of $\Delta_{i}$ and $t^{m}-1$ for all $i$.

From now on we shall assume that $V_{d}^{n}$ has only isolated singularities, i.e., $k=0$. Note that use of Zariski's theorem as above allows us to reduce the questions considered here to this special case. Let $B_{c}$ denote a small ball about a singular point $c$ of hypersurface $V_{d}^{n}$. We denote by $P_{c}$ the order of $\pi_{n}\left(B_{c}-V_{d}^{n}\right) \otimes \mathbf{Q}$ as $\mathrm{Q}\left[\pi_{1}\left(B_{c}-V_{d}^{n}\right)\right]=\mathrm{Q}\left[t, t^{-1}\right]$-module. Note that $P_{c}$ is in fact just the characteristic polynomial of the local monodromy operator of the singularity $c$. Let $T(H)$ denote a small tubular neighbourhood of $H$ in $\mathbf{C P}^{n+1}$. Denote by $P_{\infty}$ the order of $\pi_{n}\left(\partial T(H)-V_{d}^{n}\right) \otimes \mathbf{Q}$ as $\mathrm{Q}\left[t, t^{-1}\right]$-module, where the module structure is obtained from the natural homomorphism

$$
\pi_{1}\left(\partial T-V_{d}^{n}\right) \rightarrow \mathbf{Z}
$$

defined by linking with $V_{d}^{n}$.

TheOREM 2. (1) $P$ divides $\Pi P_{c}$, where the product is taken over all singular points of $V_{d}^{n}$.

(2) $P$ divides $P_{\infty}$.

EXAMPLE. Let $V_{d}^{n}$ have only singularities of Brieskorn type, i.e. which are locally given by the equation

$$
z_{1}^{p_{1}}+\cdots+z_{n+1}^{p_{n+1}}=0 .
$$

If $p_{1}, \ldots, p_{n+1}$ are pairwise relatively prime then $V_{d}^{n}$ is a $\mathbf{Q}$-manifold. The order of local $\pi_{n}$ for each singularity (3) is equal to $\prod_{0 \leqslant \alpha_{i}<p_{i}}\left(t-\omega_{1}^{\alpha_{1}} \cdots \omega_{n+1}^{\alpha_{n+1}}\right)$, where $\omega_{i}$ is a primitive root of unity of order $p_{i}$. Moreover $P_{\infty}$ is equal to

$$
\prod_{0 \leqslant \alpha_{i}<d}\left(t-\omega_{0}^{\alpha_{1}} \cdots \omega_{0}^{\alpha_{n+1}}\right)
$$


where $\omega_{0}$ is a primitive root of unity of degree $d$. In particular, Proposition 2 implies that if all $p_{i}$ 's are relatively prime to $d$ then

$$
\pi_{n}\left(\mathbf{C P}^{n+1}-\left(V_{d}^{n} \cap H\right)\right) \otimes \mathbf{Q}=0,
$$

and Proposition 3 implies that if $m$ and $d$ are relatively prime then $h^{n, 0}\left(\tilde{X}_{m}\right)=0$.

THEOREM 3. Fix $p_{1}, \ldots, p_{n+1}$ such that $\sum_{i=1}^{n+1} 1 / p_{i}<1$ and assume that all singularities of $V_{d}^{n}$ are of type (3). Then $h^{n, 0}\left(\tilde{X}_{d}\right)$ is greater than or equal to the superabundance of system of hypersurfaces of degree $d\left(1-\sum_{i=1}^{n+1} 1 / p_{i}\right)-n-2$ passing through the singularities of $V_{d}^{n}$.

Corollary. Let $q_{i}=\left(\Pi_{j=1}^{n+1} p_{j}\right) / p_{i}$ and let $V_{d}^{n}$ be given by the equation $\sum_{i=1}^{n+1} f_{q_{i}}^{p_{i}}=0$, where $f_{q_{i}}$ denotes a generic form of degree $q_{i}$. Then

$$
\pi_{n}\left(\mathbf{C P}^{n+1}-\left(V_{d}^{n} \cup H\right)\right) \otimes Q \neq 0 .
$$

Indeed, it follows from the generalized theorem of Cayley-Bacharach [4] that the superabundance in Theorem 3 is equal to 1 , and Proposition 3 implies nonvanishing of $\pi_{n} \otimes \mathbf{Q}$.

On the other hand, the following theorem gives many examples of singular hypersurfaces for which $\pi_{2}$ of the complement is trivial.

THEOREM 4. Let $V$ be a nonsingular simply connected projective manifold of dimension $n$ and let $p$ be a generic projection of $V$ into $\mathbf{C P}^{n+1}$. Then

$$
\pi_{2}\left(\mathbf{C P}^{n+1}-p(V)\right) \otimes \mathbf{Q}=0 .
$$

The proofs of these results are based on the fact that in the case $k=0$, $\pi_{n}\left(\mathbf{C P}^{n+1}-\left(V_{d}^{n} \cup H\right)\right)$ can be identified with the $n$-dimensional homology group of the infinite cyclic cover of $\mathbf{C P}^{n+1}-\left(V_{d}^{n} \cup H\right)$. It makes this $\pi_{n}$ a high-dimensional analog of the Alexander module of plane algebraic curves (cf. [3]). Theorems 2 and 3 are analogs of their counterparts for plane algebraic curves. An additional ingredient, however, is the use of the mixed Hodge structure on the homology of finite cyclic covers of $\mathbf{C P}^{n+1}-\left(V_{d}^{n} \cup H\right)$. The main step in the proof of Theorem 4 is to show that the action of

$$
\pi_{1}\left(\mathbf{C P}^{n+1}-(p(V) \cup H)\right)=\mathbf{Z}
$$

on $\pi_{2}\left(\mathbf{C P}^{n+1}-(p(V) \cup H)\right)$ is trivial. This allows us to identify the latter group with $\mathrm{H}_{2}\left(\mathbf{C P} \mathbf{P}^{n+1}-p(V) \cup H, \mathbf{Q}\right)$. Triviality of $H_{2}\left(\mathbf{C P}^{n+1}-p(V) \cup\right.$ $H, Q)$ follows from a direct homology computation.

\section{REFERENCES}

1. R. Kulkarni and J. Wood, Topology of non-singular complex hypersurfaces, Adv. in Math. 35 (1980), 239-263.

2. Lê Dũng Tráng and $\mathrm{H}$. Hamm, Un théorème de Zariski du type de Lefschetz, Ann. Sci. École Norm. Sup. (4) 6 (1973), 317-366.

3. A. Libgober, Alexander invariants of plane algebraic curves. Proc. Sympos. Pure Math., vol. 40, part 2, Amer. Math. Soc., Providence, R. I., 1983, pp. 135-143.

4. B. Segre, Some properties of differentiable varieties and transformations, Ergeb. Math. Grenzgeb. vol. 13, Springer-Verlag, 1957.

5. E. R. van Kampen, On the fundamental group of an algebraic curve, Amer. J. Math. 55 (1933).

6. O. Zariski, Algebraic surfaces, Springer-Verlag, 1971.

Department of Mathematics, University of Illinois at Chicago, Chicago, Illinois 60680 
